\title{
Residual Hearing Affects Contralateral Routing of Signals in Cochlear Implant Users
}

\author{
H. Christiaan Stronks ${ }^{a}$ Jeroen J. Briaire ${ }^{a}$ Johan H.M. Frijns ${ }^{a, b}$ \\ ${ }^{a}$ Department of Otorhinolaryngology, Leiden University Medical Center, Leiden, The Netherlands; ${ }^{b}$ Leiden Institute \\ for Brain and Cognition, Leiden University, Leiden, The Netherlands
}

\section{Keywords}

Cochlear implants · Sensorineural hearing loss · CROS .

Noise $\cdot$ Sound processing $\cdot$ Speech intelligibility

\begin{abstract}
Introduction: Contralateral routing of signals (CROS) can be used to eliminate the head shadow effect. In unilateral cochlear implant $(\mathrm{Cl})$ users, CROS can be achieved with placement of a microphone on the contralateral ear, with the signal streamed to the $\mathrm{Cl}$ ear. CROS was originally developed for unilateral $\mathrm{Cl}$ users without any residual hearing in the nonimplanted ear. However, the criteria for implantation are becoming progressively looser, and the nonimplanted ear can have substantial residual hearing. In this study, we assessed how residual hearing in the contralateral ear influences CROS effectiveness in unilateral Cl users. Methods: In a group of unilateral $\mathrm{Cl}$ users $(N=17)$ with varying amounts of residual hearing, we deployed free-field speech tests to determine the effects of CROS on the speech reception threshold (SRT) in amplitude-modulated noise. We compared 2 spatial configurations: (1) speech presented to the CROS ear and noise to the $\mathrm{Cl}$ ear $\left(\mathrm{S}_{\mathrm{CROS}} \mathrm{N}_{\mathrm{Cl}}\right)$ and (2) the reverse $\left(\mathrm{S}_{\mathrm{CI}} \mathrm{N}_{\mathrm{CROS}}\right)$. Results: Compared with the use of $\mathrm{Cl}$ only, CROS improved the SRT by $6.4 \mathrm{~dB}$ on average in the $\mathrm{S}_{\mathrm{CROS}} \mathrm{N}_{\mathrm{Cl}}$ configuration. In the $\mathrm{S}_{\mathrm{Cl}} \mathrm{N}_{\mathrm{CROS}}$ configuration, however, CROS deteriorated
\end{abstract}

karger@karger.com www.karger.com/aud

Karger $\stackrel{\text { ' }}{5}$
(C) 2021 The Author(s)

Published by S. Karger AG, Basel

This is an Open Access article licensed under the Creative Commons Attribution-NonCommercial-4.0 International License (CC BY-NC) (http://www.karger.com/Services/OpenAccessLicense), applicable to the online version of the article only. Usage and distribution for commercial purposes requires written permission. the SRT by $8.4 \mathrm{~dB}$. The benefit and disadvantage of CROS both decreased significantly with the amount of residual hearing. Conclusion: CROS users need careful instructions about the potential disadvantage when listening in conditions where the CROS ear mainly receives noise, especially if they have residual hearing in the contralateral ear. The CROS device should be turned off when it is on the noise side $\left(\mathrm{S}_{\mathrm{Cl}} \mathrm{N}_{\mathrm{CROS}}\right)$. $\mathrm{Cl}$ users with residual hearing in the $\mathrm{CROS}$ ear also should understand that contralateral amplification (i.e., a bimodal hearing solution) will yield better results than a CROS device. Unilateral Cl users with no functional contralateral hearing should be considered the primary target population for a CROS device.

(c) 2021 The Author(s)

Published by S. Karger AG, Basel

\section{Introduction}

Unilateral cochlear implant (CI) users can benefit from an intervention referred to as contralateral routing of signals (CROS). This intervention involves placement of an ear microphone contralateral to the CI ear to redirect sound to the better hearing, implanted ear. In this way, a CROS system can eliminate the head shadow effect and enhance speech understanding in silence and noise [Arora et al., 2013]. The target population for fit- 
Table 1. Subject demographics

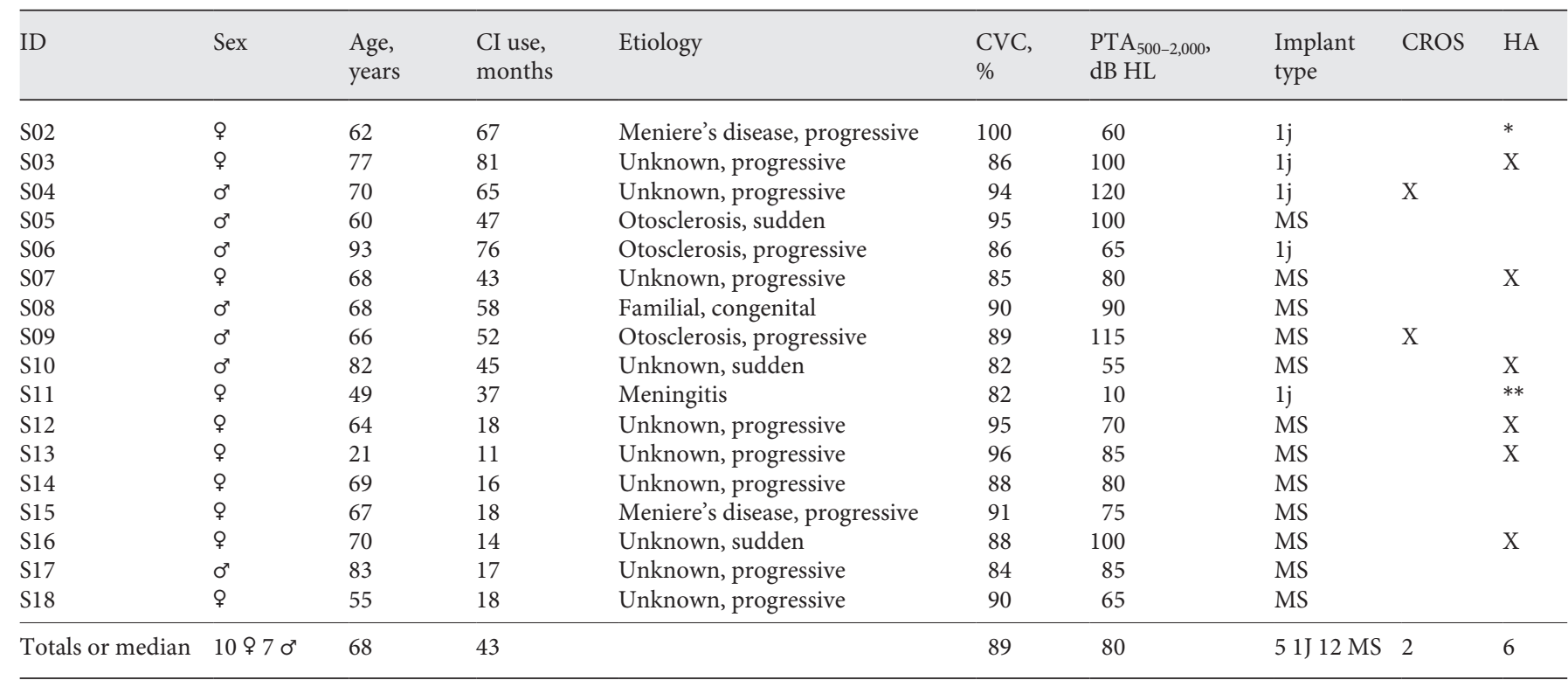

CVC, consonant-vowel-consonant phoneme score; $\mathrm{PTA}_{500-2,000}$, median pure-tone audiometric threshold across 500, 1,000, and 2,000 Hz of the nonimplanted ear; CROS, contralateral routing of signals; HA, hearing aid; CI, cochlear implant. * S02 had participated in an earlier clinical trial on bimodal hearing and had worn a HA for that study but stopped wearing it after the trial was over because of dissatisfaction with it. ${ }^{* *}$ S11 had near-normal hearing in the contralateral (CROS) ear and did not wear a HA. MS: HiRes 90K HiFocus Mid-Scala, $1 \mathrm{j}=$ HiRes 90K HiFocus $1 \mathrm{j}$.

ting a CROS device in conjunction with a CI consists of unilaterally implanted individuals without residual hearing on the contralateral side who cannot (or prefer not to) receive another implant. A CROS device is an attractive solution in countries where bilateral implantation is not the standard of care [Vickers et al., 2016]. Bilateral implantation or bimodal hearing solutions, however, are generally considered to be the treatments of choice because they restore binaural auditory input [Morera et al., 2005; Litovsky et al., 2006]. Nevertheless, CROS can significantly improve speech recognition when the target speech is coming from the front or is presented to the CROS ear. The benefits from CROS are most pronounced when listening in noise [Ernst et al., 2019] because CROS can improve the signal-to-noise ratio (SNR) by routing the speech signal to the better hearing ear. In quiet, this routing affects the sound level only in the better hearing ear because the contralateral signal is enhanced.

Advanced Bionics developed a CROS system that wirelessly streams the signal from a microphone on the side contralateral to the CI [Mosnier et al., 2019]. The system mixes the 2 signals from both devices equally with a 50:50 mixing ratio. When speech is presented at a right angle to the CROS ear and noise at a right angle to the CI ear in free field $\left(\mathrm{S}_{\mathrm{CROS}} \mathrm{N}_{\mathrm{CI}}\right)$, the maximal benefit from a CROS device equals the head shadow effect, that is, approximately $7 \mathrm{~dB}$ [Ernst et al., 2019]. When the speech and noise angles deviate from $90^{\circ}$, the benefit of CROS declines. CROS is disadvantageous when speech is presented to the CI side and noise is directed to the CROS side $\left(\mathrm{S}_{\mathrm{CI}} \mathrm{N}_{\mathrm{CROS}}\right)$ because the device mainly transmits noise to the better hearing ear and decreases the SNR in the CI ear [Taal et al., 2016].

To our knowledge, the effect of residual hearing on CROS performance has never been investigated in CI users or in other hearing solutions. Previous studies either used study participants with no appreciable residual hearing, for example, Weder et al. [2015], plugged the poorer hearing ear, for example, Van Loon et al. [2014], acknowledged residual hearing but did not account for it in the analysis, for example, Ernst et al. [2019], or did not mention residual hearing in the CROS ear at all, for example, Taal et al. [2016]. In this study, we determined the effects of residual hearing under conditions of maximal CROS benefit $\left(\mathrm{S}_{\mathrm{CROS}} \mathrm{N}_{\mathrm{CI}}\right)$ and maximal disadvantage $\left(\mathrm{S}_{\mathrm{CI}} \mathrm{N}_{\mathrm{CROS}}\right)$. To assess the effect of CROS, we performed speech-in-noise testing on study participants listening either with a CI and a CROS device or with only a CI. We recruited CI users with varying levels of residual hearing 
in the nonimplanted ear, ranging from no residual hearing at all to near-normal hearing. Of 17 participants, 6 normally wore a hearing aid in the nonimplanted ear (Table 1).

\section{Materials and Methods}

\section{Participants and Study Procedure}

This study had a prospective intervention design and was not blinded (researcher and participant were aware of the listening condition being tested), randomized (participants were selected from a database), or controlled (participants were their own control). Seventeen adults with postlingual deafness and unilaterally implanted with an Advanced Bionics CI were recruited from the Leiden University Medical Center (Table 1). Additional inclusion criteria were a CVC phoneme score of at least $80 \%$ in quiet when listening with the CI only and at least 6 months of experience with the CI. Participants were implanted with a HiRes 90K HiFocus Mid-Scala $(n=12)$ or $1 \mathrm{j}$ electrode array $(n=5)$, and all used the HiRes Optima strategy. The participants were aged 21-93 years (median: 68 years). The time after implantation ranged from 11 to 81 months (median: 43 months), and their CVC phoneme scores in quiet ranged from 82 to $100 \%$ (median: $89 \%$ ). For those who wore a hearing aid in the nonimplanted ear $(n=6)$, the hearing aid was taken out during testing, but no ear plugs were used. Two participants had been clinically fitted with a CROS device before recruitment.

Participants were fitted with a research Q90 ${ }^{\mathrm{TM}}$ processor (Advanced Bionics, Valencia, CA, USA) with their own, preferred home-use threshold and maximum comfortable levels and a Naí$\mathrm{da}^{\mathrm{TM}}$ Link CROS device (Phonak, Sonova AG, Stäfa, Switzerland). For regular home use, they each had been clinically fitted with a Tmic, an omnidirectional microphone suspended from the behind-the-ear unit of the $\mathrm{CI}$ with a rigid wire to place it just in front of the ear canal [Gifford and Revit, 2010]. The experimental speech processor used during experimentation was also fitted with a Tmic. The CROS device operates with a built-in processor microphone and thus does not feature a Tmic. We performed the testing using omnidirectional microphone settings, with all noise cancellation algorithms turned off. The acoustic filter setting was set at "standard". All participants used the "extended low" setting in their home-use device. The "standard" filter option deploys a lowpass cutoff of $350 \mathrm{~Hz}$ (on electrode 1) and a high-pass cutoff of $8,700 \mathrm{~Hz}$ (on electrode 16), whereas the "extended low" setting deploys a lower cutoff of $250 \mathrm{~Hz}$ and the same high-pass cutoff. We opted for the standard filter setting because we have used it in previous studies and wanted to be able to make direct comparison among studies (data not presented here). We did not, however, expect these filter settings to have much effect on CROS.

The median pure-tone audiogram of the nonimplanted ear for all participants is shown in Figure 1 (shaded areas indicate the interquartile range). Some audiometric pure-tone thresholds could not be determined because they exceeded the maximum stimulus level ( $>120 \mathrm{~dB})$. For this reason, we used the pure-tone median instead of the average. As a measure of functional residual hearing, we determined the median pure-tone audiometric threshold across 500, 1,000, and 2,000 Hz [Carhart, 1971]. Although this value was a median, we retained the naming convention $\mathrm{PTA}_{500-2,000}$.

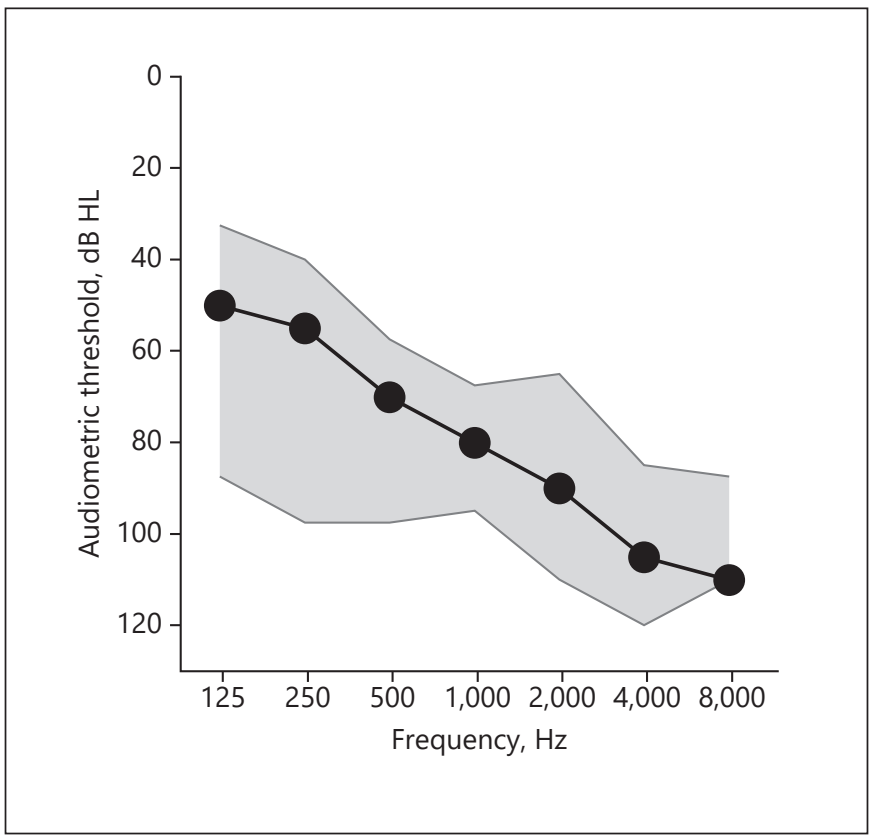

Fig. 1. Median audiogram of the nonimplanted ear with interquartile ranges $(N=17)$.

One participant (S11) had near-normal hearing in the nonimplanted ear. CROS is most effective when a speech signal is received on a deaf ear and routed to the better hearing ear. The better hearing ear of S11 was the CROS ear so that the CROS device was expected to offer little benefit for speech recognition in noise. Participant demographics are shown in Table 1.

\section{Speech-In-Noise Testing}

Speech recognition in noise was free-field tested in an audiometric, sound-attenuated booth. Participants were seated on a chair between 2 loudspeakers (MSP5A monitor speaker, Yamaha Corp., Japan) placed $1.2 \mathrm{~m}$ from the chair. We determined the effects of CROS by testing participants with a CI and CROS device (CROS condition) or without CROS (CI-only condition). The testing involved 2 spatial configurations: (1) target speech was presented to the CROS side and noise on the CI side $\left(\mathrm{S}_{\mathrm{CROS}} \mathrm{N}_{\mathrm{CI}}\right)$ and (2) the reversed configuration where speech and noise were reversed $\left(\mathrm{S}_{\mathrm{CI}} \mathrm{N}_{\mathrm{CROS}}\right)$ by turning the chair $180^{\circ}$.

To assess speech recognition in noise, we used the Flemish/ Dutch Matrix sentence material [Luts et al., 2014]. This test consists of a closed-set speech corpus of 13 combinations of 20 sentences spoken by a Flemish female. Each list was used only once per session, and the list order was randomized. The sentence order per list was not randomized. Lists 1 and 2 were used for training purposes only. Speech reception thresholds (SRTs) were measured by adaptively varying the speech level, based on a protocol described previously [Dyballa et al., 2015]. Participants listened to a sentence and repeated it out loud to the experimenter, who manually scored the correctly perceived words. We allowed guessing, and the experimenter gave no feedback. A microphone suspended from the ceiling just above the partici- 
pant ensured that the experimenter could clearly hear the participant's answers. We ran the test in a MATLAB 2017b environment (MathWorks, Inc., Natick, MA, USA). Speech recognition was tested in the presence of babble noise, consisting of temporally modulated broadband noise with spectral characteristics resembling single-talker, male speech [Stronks et al., 2020]. The babble noise was unintelligible and presented continuously throughout the tests. We obtained it from the noise material produced by the International Collegium for Rehabilitative Audiology [Dreschler et al., 2001]. The test sessions also included approximately 6 Matrix tests conducted before those described here as part of another study (data not presented). Thus, the participants were already thoroughly familiar with the Matrix test before starting the tests used in the current work.

\section{Statistics}

We used two-way repeated-measures ANOVA (2-way RM ANOVA) to test for the significance of the effects of CROS on the SRT, treating spatial configuration $\left(\mathrm{S}_{\mathrm{CI}} \mathrm{N}_{\mathrm{CROS}}\right.$ and $\left.\mathrm{S}_{\mathrm{CROS}} \mathrm{N}_{\mathrm{CI}}\right)$ and listening condition (CI or CROS) as within factors. To compare the effects of CROS in the 2 spatial configurations, we used a post hoc multiple comparisons one-sample $t$ test. Subtracting the SRT in the CROS condition from the SRT obtained with CI $\left(\mathrm{SRT}_{\mathrm{CI}}-\mathrm{SRT}_{\mathrm{CROS}}\right)$ gave the effect of CROS. We also applied a Bonferroni correction because we tested 2 conditions $\left(\mathrm{S}_{\mathrm{CI}} \mathrm{N}_{\mathrm{CROS}}\right.$ and $\left.\mathrm{S}_{\mathrm{CROS}} \mathrm{N}_{\mathrm{CI}}\right)$ against the null hypothesis $\left(\mathrm{H}_{0}\right)$ of no effect $(0 \mathrm{~dB}$ SRT difference resulting from CROS use). To this end, the calculated $P$ level was multiplied by 2 . To evaluate whether the benefit and disadvantage of CROS under the 2 spatial configurations significantly depended on residual hearing, we applied linear regression to evaluate the correlation of the effect of CROS $\left(\mathrm{SRT}_{\mathrm{CROS}}{ }^{-}\right.$ $\left.\mathrm{SRT}_{\mathrm{CI}}\right)$ with residual hearing $\left(\mathrm{PTA}_{500-2,000}\right)$. For all statistical analyses, we used GraphPad Prism v8.0.2 (GraphPad Software, San Diego, CA, USA).

\section{Results}

Speech recognition scores with and without the CROS device are shown in Figure 2a. Higher SRTs represent poorer performance. The CROS device had a statistically significant overall effect on speech recognition (2-way RM ANOVA, $F[1,16]=4.92$, and $p=0.041)$. The effect of spatial configuration was not significant $(F[1,16]=2.80$, $p=0.11$ ). As expected, the 2 factors showed a significant interaction $(F[1,16]=87.2, p<0.0001)$, with the effects of CROS depending on the spatial configuration of the signal and noise. As noted, to calculate the effects of CROS, we subtracted the SRT when using CROS from the value obtained using only the CI (SRT $\left.\mathrm{CI}-\mathrm{SRT}_{\mathrm{CROS}}\right)$. These results are shown in Figure 2b, where CROS benefits are positive and disadvantages are plotted as negative values. In the $\mathrm{S}_{\mathrm{CROS}} \mathrm{N}_{\mathrm{CI}}$ configuration, CROS yielded a significant benefit of $6.4 \mathrm{~dB}$ (1-sample, Bonferroni-corrected $t$ test against $H_{0}=0 \mathrm{~dB}$ difference, adjusted $\left.p<0.0001\right)$. In the reverse configuration $\left(\mathrm{S}_{\mathrm{CI}} \mathrm{N}_{\mathrm{CROS}}\right)$, however, CROS re- sulted in a significant disadvantage of $8.4 \mathrm{~dB}$ (adjusted $p<0.0001)$. This disadvantage was significantly greater than the advantage, by $2.0 \mathrm{~dB}$ (paired, 2-tailed $t$ test on the magnitude of the benefit and disadvantage, $t[16]=2.2$, and $p=0.041$ ).

To investigate any relation between residual contralateral hearing and the difference in magnitude of the CROS benefit and disadvantage, we plotted CROS effects under the 2 listening conditions as a function of the PTA $_{500-2,000}$ of the nonimplanted ear (Fig. 2c). The benefit of CROS (open circles) was significantly correlated with $\mathrm{PTA}_{500-2,000}$ (linear regression, $r^{2}=0.45, F[1,15]=$ 12.4 , and $p=0.0030$ ), as was the disadvantage (closed circles, $r^{2}=0.39, F[1,15]=9.8$, and $p=0.0070$ ). Results for the participant with the near-normal hearing in the CROS ear (S11) are represented by the left-most data pair (PTA $500-2,000$ of $10 \mathrm{~dB}$ ). These data carried a disproportionately large weight in the regression analysis because this participant's $\mathrm{PTA}_{500-2,000}(10 \mathrm{~dB})$ was approximately $70 \mathrm{~dB}$ better than the study population median $(83 \mathrm{~dB})$. Omitting these data from the regression analysis still yielded a significant correlation of $\mathrm{PTA}_{500-2,000}$ with the benefit $\left(r^{2}=0.25, F[1,14]=4.74\right.$, and $\left.p=0.047\right)$ but not with the disadvantage $\left(r^{2}=0.084, F[1,14]=1.28\right.$, and $p=$ $0.28)$. To visualize the differences between the 2 data sets, we plotted the difference in CROS effects (i.e., the magnitude of the benefit $\left[\mathrm{S}_{\mathrm{CROS}} \mathrm{N}_{\mathrm{CI}}\right]$ subtracted from the disadvantage $\left[\mathrm{S}_{\mathrm{CI}} \mathrm{N}_{\mathrm{CROS}}\right]$ ) as a function of $\mathrm{PTA}_{500-2,000}$ (Fig. 2d). In the figure, negative values represent participants for whom the disadvantage of CROS exceeded its benefit, as was the case in 12 of 17 participants ( $c f$. Fig. 2b). The correlation between the difference in CROS effect and residual hearing was not statistically significant however $\left(r^{2}=0.079, F[1,15]=1.3, p=0.28\right)$. Excluding participant S11 yielded a similar result $\left(r^{2}=0.11, F[1,14]=\right.$ 1.7 , and $p=0.21$ ).

In addition to the difference in magnitude between CROS benefit and disadvantage, we found a significant difference of $>5 \mathrm{~dB}$ (paired $t$ test, $t[16]=6.3$, and $p<$ 0.0001 ) between the absolute SRTs from the 2 spatial configurations when CROS was used (cf. Fig. 2a, gray bars). The average SRT was $4.6 \mathrm{~dB}$ at $\mathrm{S}_{\mathrm{CROS}} \mathrm{N}_{\mathrm{CI}}$ and $9.8 \mathrm{~dB}$ at $\mathrm{S}_{\mathrm{CI}} \mathrm{N}_{\mathrm{CROS}}$. Theoretically, these SRTs should be identical because a reversal in spatial configuration should not affect the SRT when CROS is used [Taal et al., 2016]. To investigate the contribution of residual hearing in this discrepancy, we determined the difference in the SRTs obtained in the 2 spatial configurations (the configuration effect, i.e., $\mathrm{SRT}_{\mathrm{S}_{\mathrm{CROS}} \mathrm{N}_{\mathrm{CI}}}-\mathrm{SRT}_{\mathrm{S}_{\mathrm{CI}} \mathrm{N}_{\mathrm{CROS}}}$ ) and plotted the outcomes as a function of PTA $500-2,000$ (Fig. 3). Negative 


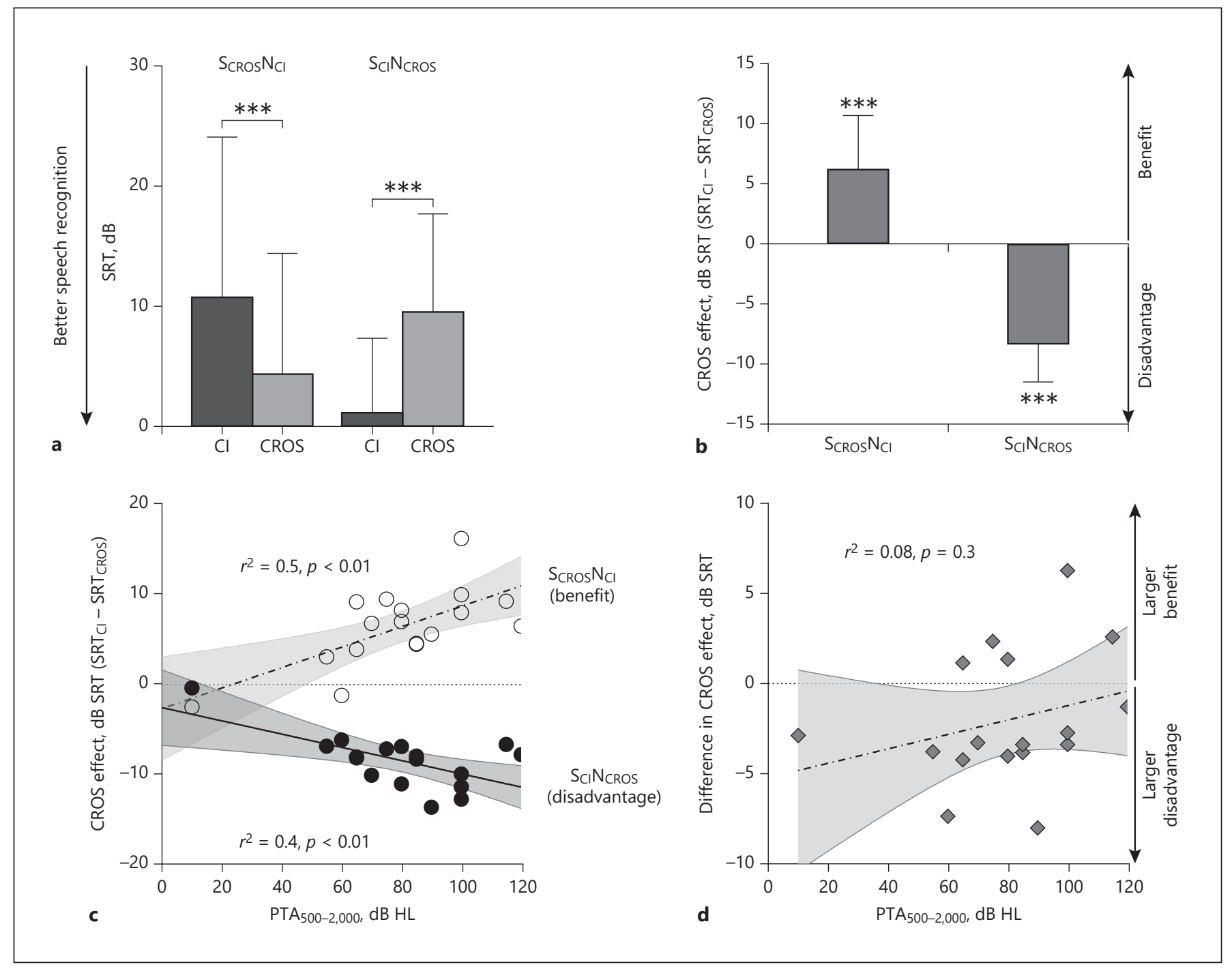

Fig. 2. Advantage and disadvantage of CROS when speech and noise are presented on opposite sides. a Absolute SRTs obtained with CI and CROS when speech was presented to the CROS ear and noise to the $\mathrm{CI}\left(\mathrm{S}_{\mathrm{CROS}} \mathrm{N}_{\mathrm{CI}}\right)$ and the reverse spatial configuration $\left(\mathrm{S}_{\mathrm{CI}} \mathrm{N}_{\mathrm{CROS}}\right)$. Lower values indicate better speech performance (arrow). b Effect of CROS on the SRT in both spatial configurations. The CROS effect was obtained by subtracting the SRT obtained with CROS from that with $\mathrm{CI}\left(\mathrm{SRT}_{\mathrm{CI}}-\mathrm{SRT}_{\mathrm{CROS}}\right)$. Positive values indicate a benefit of CROS and negative values a disadvantage (arrows). c CROS effect from (b) plotted as a function of the median pure-tone audiometric threshold across $500-2,000 \mathrm{~Hz}$ $\left(\mathrm{PTA}_{500-2,000}\right)$ of the CROS ear for $\mathrm{S}_{\mathrm{CROS}} \mathrm{N}_{\mathrm{CI}}$ (advantage, open cir-

values in this graph represent lower SRTs at $\mathrm{S}_{\mathrm{CROS}} \mathrm{N}_{\mathrm{CI}}$, that is, better speech recognition when speech was presented on the CROS ear. Because residual hearing in the implanted ear can be considered minimal, negative differences indicate a benefit of residual hearing in the CROS cles) and $\mathrm{S}_{\mathrm{CI}} \mathrm{N}_{\mathrm{CROS}}$ (disadvantage, solid circles). Thick solid and dashed lines: fitted curves using linear regression. Shaded areas: 95\% CI. d The net CROS effect calculated from (c) by subtracting the CROS effect in the $\mathrm{S}_{\mathrm{CROS}} \mathrm{N}_{\mathrm{CI}}$ configuration from that obtained in $\mathrm{S}_{\mathrm{CI}} \mathrm{N}_{\mathrm{CROS}}$. Positive values indicate a larger benefit than disadvantage and negative values a larger disadvantage (arrows). Solid line: fitted curves using linear regression. Shaded area: 95\% CI. ${ }^{* * *} p<0.0001$. CROS, contralateral routing of signals; SRT, speech reception threshold; CI, cochlear implant; 95\% CI, 95\% confidence intervals; PTA ${ }_{500-2,000}$, median pure-tone audiometric threshold across $500,1,000$, and $2,000 \mathrm{~Hz}$ of the nonimplanted ear.

ear. The linear regression showed a significant dependence of the SRT difference on the PTA $500-2,000\left(r^{2}=0.30\right.$, $F[1,15]=6.3$, and $p=0.023)$. Excluding participant S11 attenuated it to the loss of significance $\left(r^{2}=0.24\right.$, $F[1,14]=4.5$, and $p=0.051)$. 


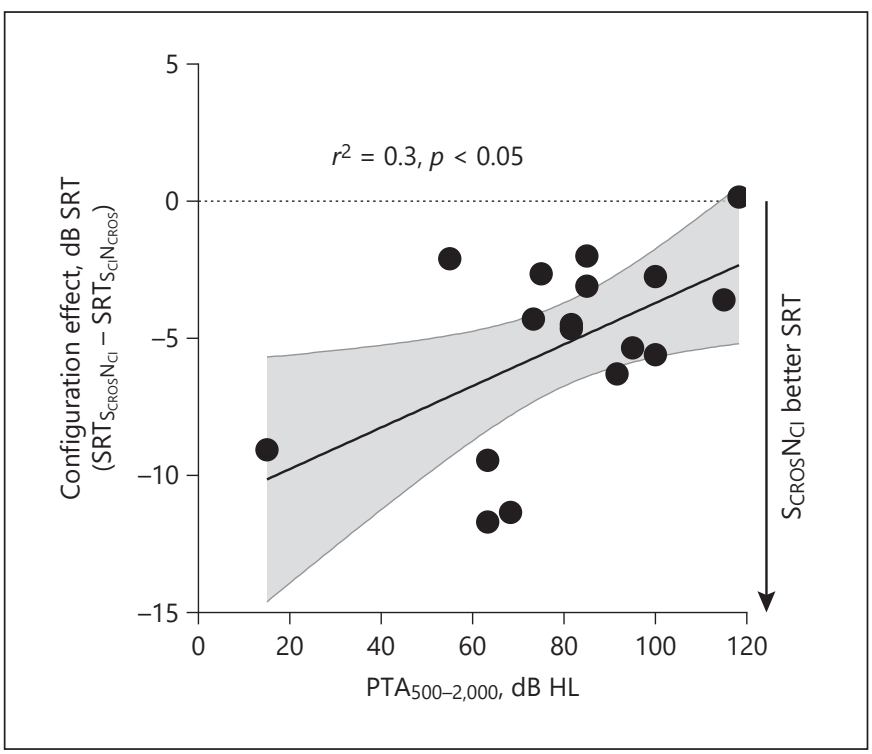

Fig. 3. Effect of the spatial configuration on the SRT as a function of residual hearing. The configuration effect was calculated by subtracting the SRT obtained when speech was presented to the CI ear and noise to the CROS ear, from the SRT obtained in the reverse configuration ( $\mathrm{SRT}_{\mathrm{S}_{\mathrm{CROS}} \mathrm{N}_{\mathrm{CI}}}-\mathrm{SRT}_{\mathrm{S}_{\mathrm{CI}} \mathrm{N}_{\mathrm{CROS}}}$ ). The CROS device was worn in both configurations. Residual hearing of the CROS ear was calculated as the median pure-tone audiometric threshold across $500-2,000 \mathrm{~Hz}\left(\mathrm{PTA}_{500-2,000}\right)$. Negative values of the configuration effect corresponded to better speech recognition when speech was presented to the CROS side. Solid line: fitted curves using linear regression. Shaded area: $95 \%$ CI. CROS, contralateral routing of signals; SRT, speech reception threshold; CI, cochlear implant; 95\% CI, 95\% confidence intervals; $\mathrm{PTA}_{500-2,000}$, median pure-tone audiometric threshold across $500,1,000$, and $2,000 \mathrm{~Hz}$ of the nonimplanted ear.

\section{Discussion}

Using 2 spatial listening configurations, we tested the benefits of a CROS device in a group of unilaterally implanted CI users with varying degrees of residual hearing. The CROS system improved speech recognition significantly, by $6 \mathrm{~dB}$, in the $\mathrm{S}_{\mathrm{CROS}} \mathrm{N}_{\mathrm{CI}}$ configuration. For $\mathrm{S}_{\mathrm{CI}} \mathrm{N}_{\mathrm{CROS}}$, however, the SRT was significantly worsened by $8 \mathrm{~dB}$ (Fig. 2b). The magnitudes of the advantage and disadvantage differed significantly. This finding was unexpected, as modeling studies have shown that the benefit $\left(\mathrm{S}_{\mathrm{CROS}} \mathrm{N}_{\mathrm{CI}}\right)$ and disadvantage $\left(\mathrm{S}_{\mathrm{CI}} \mathrm{N}_{\mathrm{CROS}}\right)$ of CROS should be equal when the mixing ratio is 50:50 [Taal et al., 2016]. Correlation analysis showed that both the benefit and disadvantage of the CROS device depended significantly on the amount of residual hearing in the CROS ear. In the $\mathrm{S}_{\mathrm{CROS}} \mathrm{N}_{\mathrm{CI}}$ configuration, the $\mathrm{CI}$ ear was the better hearing one in most participants, and we expected an overall ben- efit of CROS of $7 \mathrm{~dB}$. However, because the contralateral ear had more residual hearing, the benefit significantly decreased (Fig. 2c). We hypothesize that increasing residual hearing in the CROS ear increased its role in speech understanding because participants could become more able to use bimodal hearing. In the most extreme case of the participant with near-normal hearing in the CROS ear, the benefits of CROS were minimal (Fig. 2c). For this participant, speech was presented to the better hearing ear, and CROS improved the SNR in the poorer hearing ear with little effect on speech recognition. Conversely, we have shown that more residual hearing correlated with a lesser disadvantage of CROS in the $\mathrm{S}_{\mathrm{CI}} \mathrm{N}_{\mathrm{CROS}}$ configuration. As with the correlation between residual hearing and the benefit of CROS, we can explain this observation by the fact that the CROS ear can increasingly sustain speech recognition in noise thanks to the benefits of bimodal hearing. Based on these observations, our results indirectly show that bimodal hearing has advantages over CROS for speech recognition. Bimodal hearing is thought to improve speech recognition of CI users by providing temporal fine-structure cues, low-frequency information, and binaural cues [Qin and Oxenham, 2003; Pyschny et al., 2014].

The decreased benefit of CROS in the presence of residual hearing in the contralateral ear may have arisen from the electronic processing delay of the CROS signal. These delays are introduced by the speech processor and by the streaming of information between the CROS and the speech processor. As a result, the CROS signal presented to the CI ear is delayed relative to the acoustic signal in the CROS ear. With an oscilloscope (SmartScope, LabNation, Antwerp, Belgium) and a custommade dummy implant, we have determined that the processing delay of the $\mathrm{CI}$ and CROS devices adds up to approximately $55 \mathrm{~ms}$. This delay must be corrected for the 1-ms delay imposed by the mechanotransduction process by hair cells [Temchin et al., 2005] and for the traveling wave time, which is approximately $7 \mathrm{~ms}$ at 150 $\mathrm{Hz}$ and $<1 \mathrm{~ms}$ at frequencies $>4 \mathrm{kHz}$ [Ruggero and Temchin, 2007]. The net delay between the electrical and acoustical speech signals can thus be estimated at approximately $47-53 \mathrm{~ms}$. This interaural delay is sufficiently large to disrupt speech recognition [Wess et al., 2017] and may result in the perception of echoes [Litovsky et al., 1999].

Modeling work [Taal et al., 2016] has shown that the advantage and disadvantage of the CROS system are maximal and approximately symmetrical when the speech and noise are presented on opposite ears. The reported symmetry, however, holds only under the as- 
sumption that the CROS ear has no residual hearing. In the model of Taal et al. [2016], the advantage in this scenario was $10.4 \mathrm{~dB}$, and the disadvantage in the reverse scenario was $9.9 \mathrm{~dB}$. These values match reasonably well our interpolated values of approximately $11 \mathrm{~dB}$ at a PTA $_{500-2,000}$ of $120 \mathrm{~dB}$ (Fig. 2c). For this reason, the trend in Figure 2d may be realistic because it indeed approaches the point of equal benefit and disadvantage at a PTA $_{500-2,000}$ of $120 \mathrm{~dB}$ HL (an approximately $11 \mathrm{~dB}$ benefit and disadvantage). However, the difference between CROS benefit and disadvantage in the 2 spatial configurations $\left(\mathrm{S}_{\mathrm{CROS}} \mathrm{N}_{\mathrm{CI}}\right.$ and $\left.\mathrm{S}_{\mathrm{CI}} \mathrm{N}_{\mathrm{CROS}}\right)$ did not correlate significantly with the amount of residual hearing. Based on the current data, therefore, we can conclude that (1) more residual hearing is significantly associated with less benefit and a smaller disadvantage (Fig. 2c) and (2) in a group of participants with different levels of residual hearing, the overall disadvantage significantly exceeds the benefit (Fig. 2b). We cannot, however, deduce how the discrepancy between the benefit and disadvantage depends on residual hearing (Fig. 2d).

The values shown in Fig. $2 b-d$ were obtained by arithmetic summation of SRTs, which increases the random errors. Our results, thus, should be interpreted with caution. In addition, the linear fits applied for the benefit and disadvantage of CROS in Figure $2 \mathrm{c}$ are in reality nonlinear curves because floor and ceiling effects are expected at the extreme ends of the $\mathrm{PTA}_{500-2,000}$ range. To achieve more realistic, nonlinear curve fits would require more participants with relatively good residual hearing $\left(\right.$ PTA $\left._{500-2,000}<50 \mathrm{~dB}\right)$ and without functional hearing $\left(\right.$ PTA $_{500-2,000}>100 \mathrm{~dB}$ ).

Residual hearing could also explain the finding that absolute SRTs obtained with the CROS device were substantially higher (i.e., speech recognition was worse) in the $\mathrm{S}_{\mathrm{CI}} \mathrm{N}_{\text {CROS }}$ than in the $\mathrm{S}_{\mathrm{CROS}} \mathrm{N}_{\mathrm{CI}}$ configuration (Fig. 3). As noted, speech recognition would be expected to be identical between the 2 configurations when a CROS device is worn with a mixing ratio of 50:50 [Taal et al., 2016]. The most likely explanation is that the residual hearing in our participant group substantially improved speech recognition, which would have been most noticeable in the $\mathrm{S}_{\mathrm{CROS}} \mathrm{N}_{\mathrm{CI}}$ condition. The relatively pronounced effects we found in this study can be explained by the fact that the spatial configurations tested were the most extreme cases. For $\mathrm{S}_{\mathrm{CROS}} \mathrm{N}_{\mathrm{CI}}$, the SNR in the CROS ear and the benefit of residual hearing on speech recognition are maximal. Conversely, for $\mathrm{S}_{\mathrm{CI}} \mathrm{N}_{\mathrm{CROS}}$, the disadvantage of residual hearing will be maximal, given that the SNR is at its lowest on the CROS ear.

Effect of Residual Hearing on CROS
Our findings must be placed in perspective. The indication for a CROS device is a unilateral implant with no residual hearing in the nonimplanted ear. In our study population, however, a substantial number of participants had residual hearing. Of the 17 included participants, 6 normally wore a hearing aid in the contralateral ear, referred to as bimodal hearing. The criteria for a bimodal fitting are not strictly defined, but some authors say that almost all unilateral CI users can benefit from a contralateral hearing aid, even when the nonimplanted ear has severe (65-79 $\mathrm{dB}$ ) to profound (80-94 dB) hearing loss [Ching, 2005]. For this reason, many of our participants might be preferably fitted with a hearing aid rather than a CROS device.

Limitations of this study include the fact that all but one of the participants had relatively poor residual hearing. As a consequence, significance testing of the correlations between the various outcome measures and residual hearing depended disproportionately on a single participant (S11) with near-normal hearing in the CROS ear (Fig. 2c, d, 3). The benefit of CROS still significantly de-

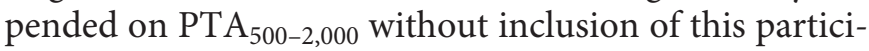
pant, but the association with the disadvantage did not remain significant (Fig. 2c). Thus, the correlation between PTA ${ }_{500-2,000}$ and the disadvantage of CROS should be interpreted with some caution. Likewise, with exclusion of data for participant S11, the discrepancy between absolute SRTs was no longer associated significantly $(p=$ 0.051) with $\mathrm{PTA}_{500-2,000}$ in either spatial configuration when listening with CROS (Fig. 3). The influence of the Tmic, if any, is unclear and requires further study.

\section{Conclusion}

Using 2 opposing spatial configurations $-\mathrm{S}_{\mathrm{CROS}} \mathrm{N}_{\mathrm{CI}}$ and $\mathrm{S}_{\mathrm{CI}} \mathrm{N}_{\mathrm{CROS}}$ - we have shown that the benefit and disadvantage of CROS are maximal in the absence of functional residual hearing. Both the benefit and disadvantage decrease with increasing residual hearing, but the disadvantage always outweighs the benefit in these conditions. These findings point to the need to disable CROS when speech is coming from the CI side and noise from the other side because the disadvantage may outweigh any benefit. Professional health-care providers administering CROS devices should carefully advise their clients of these considerations, especially when residual hearing is present. In future applications, automated SNR analysis is worth pursuing as a way to switch off CROS functionality when the SNR is better on the CI side.

Audiol Neurotol 2022;27:75-82

DOI: $10.1159 / 000515210$ 


\section{Acknowledgements}

We thank the participants for their time and dedication and Raphael Koning (Advanced Bionics European Research Center, Hannover, Germany) for technical support.

\section{Statement of Ethics}

This study adhered to the tenets of the Helsinki declaration [World Medical Association, 2013] and was approved by the Institutional Review Board of the Leiden University Medical Center (P02.106/P02.0778L). All participants provided written informed consent before participating in the study.

\section{Funding Sources}

This study was financially supported by Advanced Bionics, LLC (Valencia, CA, USA).

\section{Conflict of Interest Statement}

The authors have no other funding, financial relationships, or conflicts of interest to disclose.

\section{Author Contributions}

H.C.S.: I.R.B. approval, experimental design, data collection, analyses, and draft writing. J.J.B. and J.H.M.F.: experimental design, intellectual contributions, and critical revisions of the first draft.

\section{References}

Arora R, Amoodi H, Stewart S, Friesen L, Lin V, Nedzelski J, et al. The addition of a contralateral routing of signals microphone to a unilateral cochlear implant system: a prospective study in speech outcomes. Laryngoscope. 2013 Mar; 123(3):746-51.

Carhart R. Observations on relations between thresholds for pure tones and for speech. J Speech Hear Disord. 1971 Nov;36(4):476-83.

Ching TYC. The evidence calls for making binaural-bimodal fittings routine. Hear J. 2005 Nov;58(11):32-41.

Dreschler WA, Verschuure H, Ludvigsen C, Westermann S. ICRA noises: artificial noise signals with speech-like spectral and temporal properties for hearing instrument assessment. International collegium for rehabilitative audiology. Audiology. 2001 May-Jun; 40(3):148-57.

Dyballa KH, Hehrmann P, Hamacher V, Nogueira W, Lenarz T, Büchner A. Evaluation of a transient noise reduction algorithm in $\mathrm{CO}^{-}$ chlear implant users. Audiol Res. 2015 Jun 11; 5(2):116.

Ernst A, Baumgaertel RM, Diez A, Battmer RD. Evaluation of a wireless contralateral routing of signal (CROS) device with the advanced bionics Naída CI Q90 sound processor. Cochlear Implants Int. 2019 Jul;20(4):182-9.

Gifford RH, Revit LJ. Speech perception for adult cochlear implant recipients in a realistic background noise: effectiveness of preprocessing strategies and external options for improving speech recognition in noise. J Am Acad Audiol. 2010 Jul-Aug;21(7):441-8. quiz 87-8.
Litovsky RY, Colburn HS, Yost WA, Guzman SJ. The precedence effect. J Acoust Soc Am. 1999 Oct;106(4 Pt 1):1633-54

Litovsky RY, Johnstone PM, Godar SP. Benefits of bilateral cochlear implants and/or hearing aids in children. Int J Audiol. 2006 Jan; 45(Suppl 1 1):S78-91.

Luts H, Jansen S, Dreschler W, Wouters J. Development and normative data for the Flemish/ Dutch matrix test. Belgium and Amsterdam The Netherlands: Katholieke universiteit Leuven and Academic Medical Center; 2014. Unpublished article.

Morera C, Manrique M, Ramos A, Garcia-Ibanez L, Cavalle L, Huarte A, et al . Advantages of binaural hearing provided through bimodal stimulation via a cochlear implant and a conventional hearing aid: a 6 -month comparative study. Acta Otolaryngol. 2005 Jun;125(6): 596-606.

Mosnier I, Lahlou G, Flament J, Mathias N, Ferrary E, Sterkers O, et al . Benefits of a contralateral routing of signal device for unilateral Naída CI cochlear implant recipients. Eur Arch Otorhinolaryngol. 2019 Aug;276(8): 2205-13.

Pyschny V, Landwehr M, Hahn M, Lang-Roth R, Walger M, Meister H. Head shadow, squelch, and summation effects with an energetic or informational masker in bilateral and bimodal CI users. J Speech Lang Hear Res. 2014; 57(5):1942-60.

Qin MK, Oxenham AJ. Effects of simulated cochlear-implant processing on speech reception in fluctuating maskers. J Acoust Soc Am. 2003;114(1):446-54

Ruggero MA, Temchin AN. Similarity of traveling-wave delays in the hearing organs of humans and other tetrapods. J Assoc Res Otolaryngol. 2007;8(2):153-66.
Stronks HC, Briaire JJ, Frijns JHM. The Temporal Fine Structure of Background Noise Determines the Benefit of Bimodal Hearing for Recognizing Speech. J Assoc Res Otolaryngol. 2020;21(6):527-544.

Taal CH, van Barneveld DC, Soede W, Briaire JJ, Frijns JH. Benefit of contralateral routing of signals for unilateral cochlear implant users. J Acoust Soc Am. 2016 Jul;140(1):393-401.

Temchin AN, Recio-Spinoso A, van Dijk P, Ruggero MA. Wiener kernels of chinchilla auditory-nerve fibers: verification using responses to tones, clicks, and noise and comparison with basilar-membrane vibrations. J Neurophysiol. 2005 Jun;93(6):3635-48.

Van Loon MC, Goverts ST, Merkus P, Hensen EF, Smits C. The addition of a contralateral microphone for unilateral cochlear implant users: not an alternative for bilateral cochlear implantation. Otol Neurotol. 2014 Oct;35(9): e233-9.

Vickers D, De Raeve L, Graham J. International survey of cochlear implant candidacy. Cochlear Implants Int. 2016 Apr;17(Suppl 1):36-41.

Weder S, Kompis M, Caversaccio M, Stieger C. Benefit of a contralateral routing of signal device for unilateral cochlear implant users. Audiol Neurootol. 2015 Apr;20(2):73-80.

Wess JM, Brungart DS, Bernstein JGW. The effect of interaural mismatches on contralateral unmasking with single-sided vocoders. Ear Hear. 2017 May-Jun;38(3):374.

World Medical Association. World medical association declaration of helsinki: ethical principles for medical research involving human subjects. JAMA. 2013 Nov;310(20):2191-4. 